\title{
A ROBUST ACCESS PROTOCOL FOR WIRELESS SENSOR NETWORKS
}

\author{
Chung Shue Chen ${ }^{\dagger *}$, Wing Shing Wong* \\ * Department of Information Engineering, The Chinese University of Hong Kong, Shatin, Hong Kong \\ $\dagger$ LORIA-CNRS, Rue du Jardin Botanique, 54600 Villers Les Nancy, France \\ Email: cschen@ieee.org, wswong@ie.cuhk.edu.hk
}

\begin{abstract}
This paper introduces a family of periodic binary sequences with interesting cross-correlation properties for potential applications in distributed wireless accessing. Following Massey's model of collision channel without feedback, a simple and robust multiple access protocol that does not require complicated processing such as back-off algorithms or random number generation is proposed for wireless sensor and ad hoc networks. In addition, energy-efficient data reception is achievable by the protocol while continuous channel listening can be eliminated. Besides, a simple predictive collision avoidance scheme is presented for the enhancement of system throughput. Simulation studies are carried out to show its effectiveness.
\end{abstract}

\section{INTRODUCTION}

Technological advances in recent years enable the fabrication of miniaturized devices or sensors with built-in information processing for data communications. Building these devices into a coordinated network of sensing or controlling units could offer potentials for many innovative applications. However, these individual devices usually have quite limited processing and communication capabilities. Meanwhile, they have strict energy constraints. Existing protocols can be generally categorized into contention-based and TDMA-based solutions $[1,2]$. When considering systems with dynamic network topology, sharing a radio channel among a large number of devices with the requirement of well-coordinated transmissions and time offsets could be very complicated particularly to thin devices.

For these reasons, it is desirable to have a simple multiple access protocol that does not require frequent monitoring of the channel for feedback information and can avoid complicated processing such as back-off algorithm or random number generation. This will provide an alternative for applications with strict system limitations. Designing such a network protocol can be traced back to Massey's work of collision channel without feedback [3] and the literature [4-7]. Some related works of binary sequence design and analysis for different communication applications can be found in [8-12].

Here, we focus on the recent development of a new

The work described in this paper was supported by a grant from the Research Grants Council of the Hong Kong Special Administrative Region (Project no. CUHK416906). The work was carried out when Chung Shue Chen was with the Department of Information Engineering, The Chinese University of Hong Kong, Shatin, Hong Kong. family of periodic binary sequences, namely wobbling sequences [13], and its applications. These sequences have nice cross-correlation properties that make them suitable for defining multiple access protocols that do not require a feedback link. They can be easily generated and implemented. At the same time, each individual user can operate in a relatively distributed manner after an allocation of sequence key with respect to its requested transmission rate. However, the current design does not have a single peak auto-correlation property for sequence numbering acquisition. For the simplicity of operation, here we assume each data packet will contain a header of user identification and sequence number in a common practice.

The rest of the paper is organized as follows. The system model is presented in Section II. The access protocol and resultant properties are described accordingly. A numerical analysis of the system performance is presented in Section III. For channel utilization efficiency, a collision avoidance scheme has been investigated in Section IV. A concluding remark is drawn in Section V.

\section{SySTEM MODEL}

Following Massey's model of collision channel without feedback [3], a communication channel is divided into time slots with equal duration. It is assumed that the channel is shared by $M$ active users and each user follows a binary protocol sequence, $W=\{W(0), W(1), \cdots\}$. An active user transmits a packet at time slot $i$ if and only if $W(i)=1$. Otherwise, it will be in an idle state. We assume the users know and align to the slot boundaries. However, users are not required to synchronize each other.

At any time slot, if more than one user transmit, the receiver is assumed to detect a collision and all transmitted packets in that time slot will be lost. On the other hand, if only one user transmits, it is assumed that the receiver can receive the packet correctly. Discussions on advanced coding and decoding schemes can be found in [3]. One simple approach to identify the sender is to require each packet to include a header that contains user identity and data sequence number similar to that in ALOHA. If the payload of a packet is large enough, the cost of overhead could be quite small.

The effective rate of a user is defined as the fraction of packets it can send without suffering any collision. In the literature, many studies have focused on designing sequences that can provide high sum rate as well as support a large number of users [3-7]. In a random access system, one is usually most interested in the symmetric 
case in which all users are signaling at the same rate [3]. In this case, the capacity or effective sum rate of a slotsynchronized system will approach to $e^{-1}$ as the number of active users, $M$, goes to infinity.

In addition to the system throughput, it is also important to look at the channel reliability for each individual user. It is favorable to have a design of protocol sequences which can provide reliability and robustness such that one can always ensure the availability of a communication channel for any of the active users as long as the sum of requested rates does not exceed a specific bound. In the following, we will describe the details of wobbling sequences and their properties for wireless multiple access.

\section{A. Linear Congruence Sequences}

Let $W=\{W(i), i=0,1,2, \cdots\}$ be a binary sequence. The sequence $W$ can also be represented by indexing the positions at which it has value 1 , i.e., by

$$
\left\{I_{W}(i), i=1,2, \cdots\right\}
$$

where $I_{W}(i)$ denotes the position at which the $i$-th entry of 1 's in $W$ appears.

For a periodic binary sequence with a minimum period $L$, following [3], its duty factor is defined by

$$
r=\frac{1}{L} \sum_{i=0}^{L-1} W(i) .
$$

Let $b$ and $l$ be two relatively prime integers with $0 \leq b<$ $l$. A linear congruence sequence [8] generated by $(b, l)$ can be defined by

$$
I_{W}(i)=i l+i b-\left\lfloor\frac{i b}{l}\right\rfloor l
$$

where the integer $b$ is known as the key generator of the sequence [13]. It has a duty factor of $1 / l$. It is cyclic and has a minimum period of $l^{2}$ for $b>0$. Note that, when $b=0,(3)$ can be written as

$$
I_{W}(i)=i l \text {. }
$$

The sequence has a period of $l$.

When $l$ is prime, the above sequence is also known as a prime sequence [9]. One may define the sub-sequence

$$
W=\left\{W(i), i=0,1, \cdots, l^{2}-1\right\}
$$

as the core pattern of a linear congruence sequence.

For example, the sequence $W$ generated by $(b=2, l=$ 3 ) has a period of 9 . The core pattern is given by $\{0,0,0,0,1,0,1,0,1\}$, while $I_{W}=\{5,7,9, \cdots\}$. Its duty factor is equal to $1 / 3$.

\section{B. Wobbling Sequences}

Denote the linear congruence sequence generated by $(b, l)$ by $S_{b, l}$ and let $\boldsymbol{L}$ represent the operator that shifts a sequence by one element to the left. Therefore,

$$
\boldsymbol{L} S(i)=S(i+1) \text {. }
$$

In the following, the discussion will focus on the case in which

$$
l=p^{i}
$$

for prime $p$ and integer $i$, such that $l$ is the power of a prime number. Consequently, we have the following definitions.

Definition 1 [13] Let $l=p^{i}$, with $i \geq 2$, be a prime power and $b$ be an integer satisfying $0<b<l$. For $1 \leq d<l$, the wobbling sequence generated by $(b, l)$ with duty factor $d / l, W_{b, l, d}$, is defined by

$$
\begin{aligned}
W_{b, l, d}(i)=S_{b, l}(i) \vee \boldsymbol{L}^{l} S_{b, l}(i) \vee & \boldsymbol{L}^{2 l} S_{b, l}(i) \vee \cdots \\
& \vee \boldsymbol{L}^{(d-1) l} S_{b, l}(i)
\end{aligned}
$$

where $\vee$ is the binary operator defined by

$$
\left\{\begin{array}{l}
0 \vee 0=0 \\
0 \vee 1=1 \vee 0=1 \vee 1=1
\end{array}\right.
$$

For $b=0, W_{b, l, d}$ is defined by

$$
\begin{array}{r}
W_{b, l, d}(i)=S_{b, l}(i) \vee \boldsymbol{L}_{b, l}(i) \vee \\
\boldsymbol{L}^{2} S_{b, l}(i) \vee \cdots \\
\vee \boldsymbol{L}^{d-1} S_{b, l}(i) .
\end{array}
$$

The duty factor of the above sequence $W_{b, l, d}$ is equal to $d / l$, while the minimum period is equal to $l^{2}$. For example, given $p=3$ and $l=p^{2}$, the $I_{W}$ representation of $S_{(b=1, l=9)}$ is given by

$$
(10,20,30,40,50,60,70,80,81, \cdots)
$$

while $\boldsymbol{L}^{9} S_{1,9}$ is consequently expressible as

$$
(1,11,21,31,41,51,61,71,72, \cdots)
$$

by a shift of (11) toward the left by nine elements as defined by (6). The wobbling sequence $W_{1,9,3}$ can be denoted by its counterpart $I_{W}$ which is given by

$$
\begin{array}{r}
(1,2,10,11,12,20,21,22,30,31,32,40,41,42,50 \\
51,52,60,61,62,63,70,71,72,73,80,81 \cdots)
\end{array}
$$

following (8). Its duty factor is equal to $1 / 3$ and the minimum period is 81 .

\section{Construction of the Sequence Family}

Based on Definition 1, a family of wobbling sequences which can accommodate a large number of active users can be formulated in the following way [13]. It also supports sequences with different duty factors for the purpose of multirate transmissions.

Definition 2 For any prime $p$ and $0<d \leq p$, construct $a$ family of binary codes, $\mathbf{F}_{p}(d)$, as follows:

1. The wobbling sequence generated by $\left(b=0, l=p^{2}\right)$ is in $\mathbf{F}_{p}(d)$.

2. For $i=1,2, \cdots$, let $j=2^{i}$. For $0<b<p$, the wobbling sequence $W_{b, p^{j}, d}$ is in $\mathbf{F}_{p}(d)$. 
Consider an application of a finite subset of the code set $\mathbf{F}_{p}(d)$ as protocol sequences, as long as the sum of duty factors does not exceed one, i.e.,

$$
\sum_{j=1}^{M} r_{j} \leq 1,
$$

it can be shown [13] that for any user $i$, a minimum number of

$$
K r_{i}^{2} / p
$$

transmission packets will not be blocked for this user within the common period encountered, says $K$ slots. This unsuppressibility property ensures that all sensors can successfully transmit information to a guaranteed amount in every time period.

For example, in the case that requested rates of users are symmetric such that

$$
r_{i}=1 / p
$$

for each user $i$, one can let $d=p$ and employ wobbling sequences generated by $\left(b, p^{2}\right)$, where $b=\{0,1,2, \cdots, p-$ 1\}. Here,

$$
K=p^{4} .
$$

As a result, we have a group of $p$ different sequences with respect to different $b$. For each user, a number of at least $p$ un-blocked slots are guaranteed for every cycle of $p^{4}$ slots. Based on the cross-correlation properties of wobbling sequences, a lower bound on the sum of effective rates of allowable users in the system can be derived [13]. A numerical study on the system and individual throughputs of wobbling sequence based protocols in the model of collision channel without feedback is presented in the next section.

\section{PERFORMANCE EVAluAtion}

An investigation on the effective rates of users is carried out. Both individual and system throughputs have been measured. We assume that there are $M$ active users and each has a requested rate $1 / p$ of the channel bandwidth. Their protocol sequences are used to specify their channel access time. Accordingly, a user will have a permission to transmit at a corresponding time slot if its protocol sequence refers to ' 1 '. Otherwise, it will be in an idle state.

To construct the scheme, we follow Definition 1. Let $l=p^{2}$ and $d=p$. The duty factor is equal to $d / l$, i.e., $1 / p$. By (8) and (10), a number of $p$ wobbling sequences with

$$
b=\{0,1,2, \cdots, p-1\}
$$

are generated respectively. Since users are unsynchronized to each other, there will be a relative time shift between any two protocol sequences. For example, users may have different starting time. In simulations, we assume that the relative time shift between two protocol sequences is uniformly distributed in the encountered minimum period. In

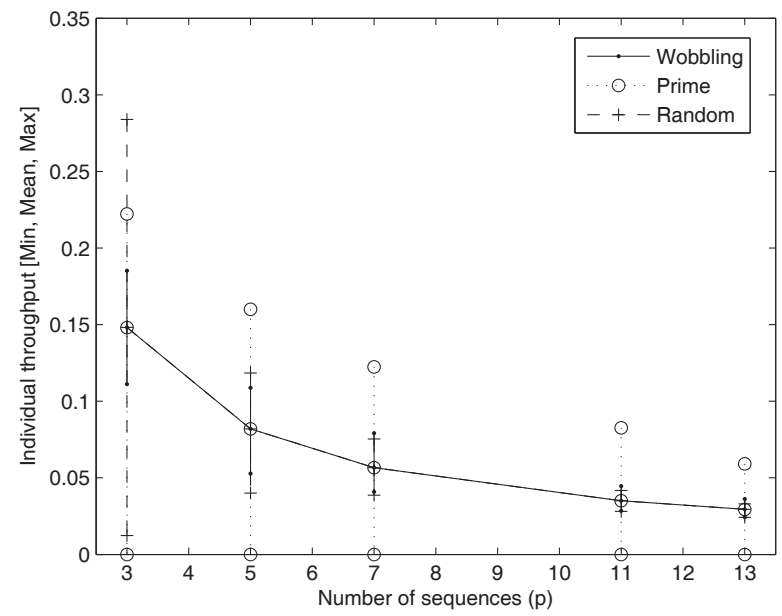

Figure 1: The minimum, mean and maximum individual throughputs of users $\left(r_{i}=1 / p\right)$ from simulation are shown.

the above case, it is in the range of $\left[0, p^{4}-1\right]$. One can find that the number of combinations of relative time shifts among all the protocol sequences can be very large.

First, we observe the user throughputs (or effective rates) when the system is fully saturated, i.e.,

$$
\sum_{i=1}^{M} r_{i}=1
$$

where $M=p$ and $r_{i}=1 / p$. Fig. 1 shows the results of individual throughputs of users with respect to different $p$ by a simulation of $10^{6}$ runs. The minimum, mean and maximum obtained from wobbling sequence are plotted and compared with those from prime sequence and a random access respectively. A prime sequence is defined by (3), while a random access here refers to the result of channel permissions which are determined by drawing with a probability of the desired duty factor $r_{i}$ from a uniform distribution.

Comparing the performance of wobbling and prime sequences, the means of their individual throughputs are almost equal. However, note that a user with prime sequence could be completely blocked by other active users in the system as shown in Fig. 1. Meanwhile, wobbling sequence has demonstrated its robustness that there cannot have enough collisions to completely block any particular sequence no matter how they are shifted with respect to one another as long as (14) is satisfied. It eliminates the risk of being completely blocked. The range of individual throughputs from simulation is indicated by [Min, Max], while the standard deviation is given in Fig. 2 in order to reflect the degree of fluctuation. Both Fig. 1 and Fig. 2 show that the wobbling sequence has a much more stable performance than the prime sequence. However, it should be noted that the prime sequences have a shorter sequence period by definition, i.e., $p^{2}$.

The effective rate of a user in the aforementioned random access is measured in a length of $p^{4}$ with reference to the 


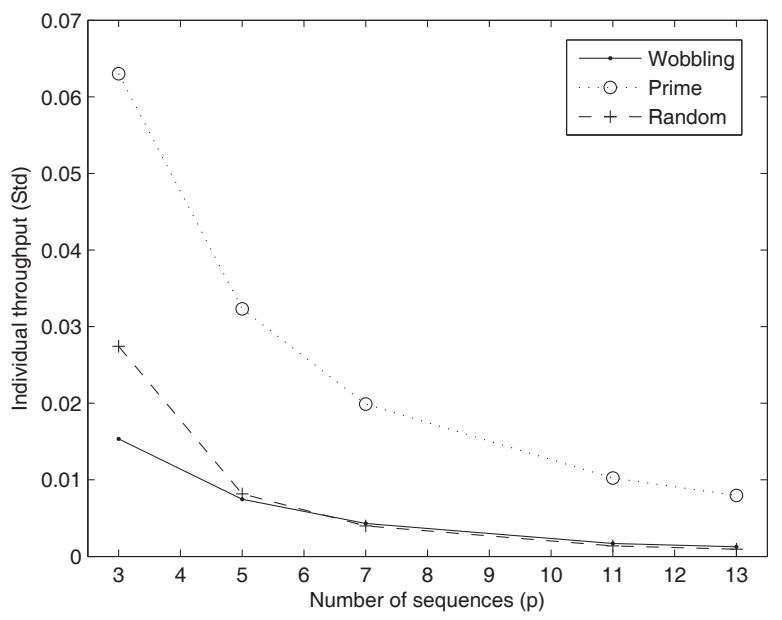

Figure 2: The standard deviation of individual throughputs of users is shown. The result is associated with Fig. 1.

common period encountered here by wobbling and prime sequences. As shown in Fig. 1 and Fig. 2, the performance of random access has quite similar behavior to that of wobbling sequences, especially when $p$ is relatively large. It is reasonable that the means of the three schemes are almost the same after taking averages over the runs with respective to the uniformly distributed relative time shifts. As shown in Fig. 1, the wobbling sequence can guarantee a higher individual minimum throughput in general. However, the difference is insignificant for $p \geq 7$ when comparing with the random access. Also, it should be noted that a user in the random access has always a non-zero probability of being completely blocked. The probability would depend on the period considered.

Fig. 3 shows the system throughputs. It indicates similar phenomena as those occurred in Fig. 1. Besides, the system throughput will tend to $e^{-1}$ as $p$ goes to infinity. In addition to the mean value, Fig. 4 shows the standard deviation of system throughputs. By Fig. 1-4, one can find that the wobbling sequences can provide a very robust and stable service. Meanwhile, it guarantees the non-totally-blockage for every user. In addition to the above studies in symmetric rates, two case studies on multi-rate transmissions are conducted. The result is included in the Appendix for reference.

Furthermore, we have carried out a study on the system performance in different traffic loads. In the simulation, we assume that in any time instant there are at most $p$ active users and each of them transmits following the wobbling sequences with duty factor $1 / p$ over a fixed duration of the sequence period $p^{4}$. It is modeled as a number of $p$ arrival processes, which correspond to the number of deployed wobbling sequences in the system. The idle time between two active users in a process is assumed to have an exponential distribution. Fig. 5 shows the system throughputs for $p=3,5$ and 7 . The offered traffic is defined as the

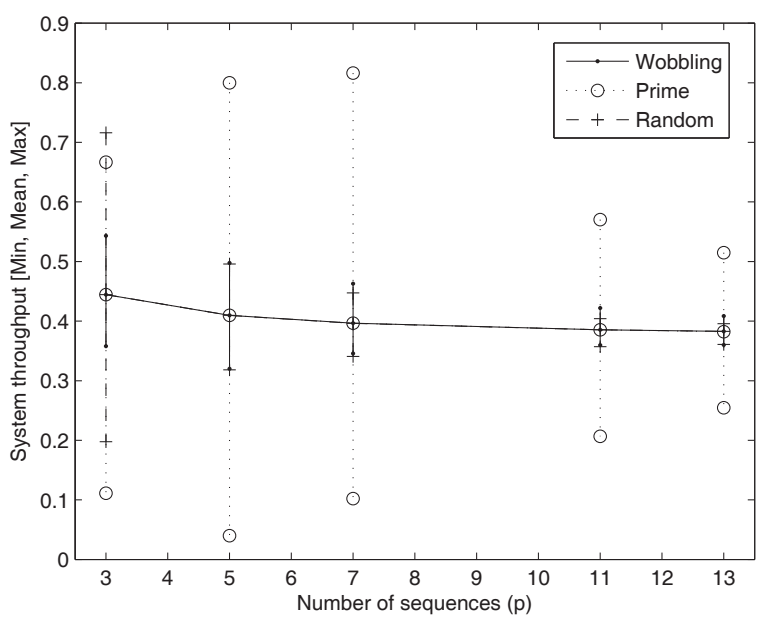

Figure 3: The system throughput is plotted with respect to different prime $p$.

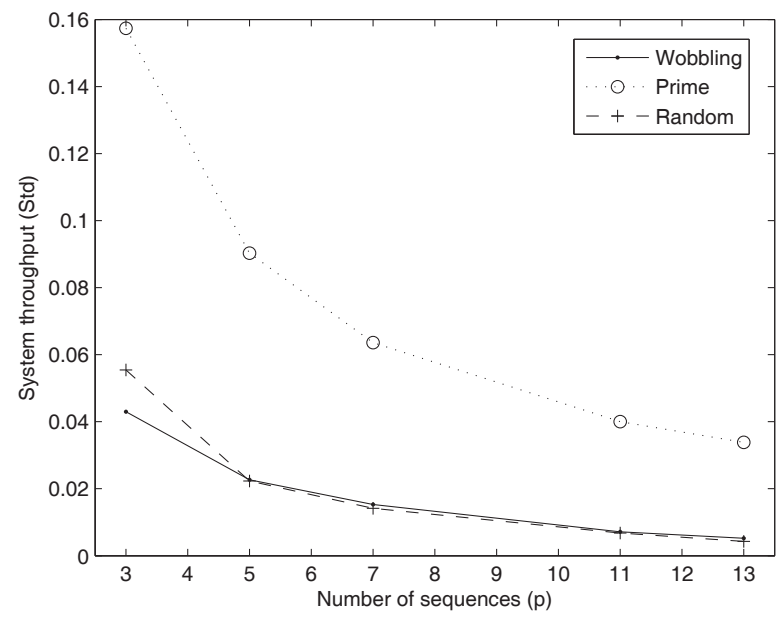

Figure 4: The standard deviation of system throughputs is shown. The result is associated with Fig. 3.

average number of packets generated (or arrived) to the system per transmission time. The result shows similar characteristics with respect to slotted ALOHA.

To draw a summary, the wobbling sequence has the following major advantages. In contrast to the other, these sequences hold the feature of non-totally-blockage and can guarantee the availability of a communication channel for every user. Secondly, this robust access protocol does not require complicated processing of random number generation. Thirdly, as wobbling sequences are deterministic, a receiver does not need to continuously listen the channel in data reception. Once a packet is successfully received, the receiver can explicitly address to its coming packets since the transmitter's wobbling sequence can be identified or reconstructed simply based on the information of $(b, l, d)$ and sequence index in a header. Such an operation could be 


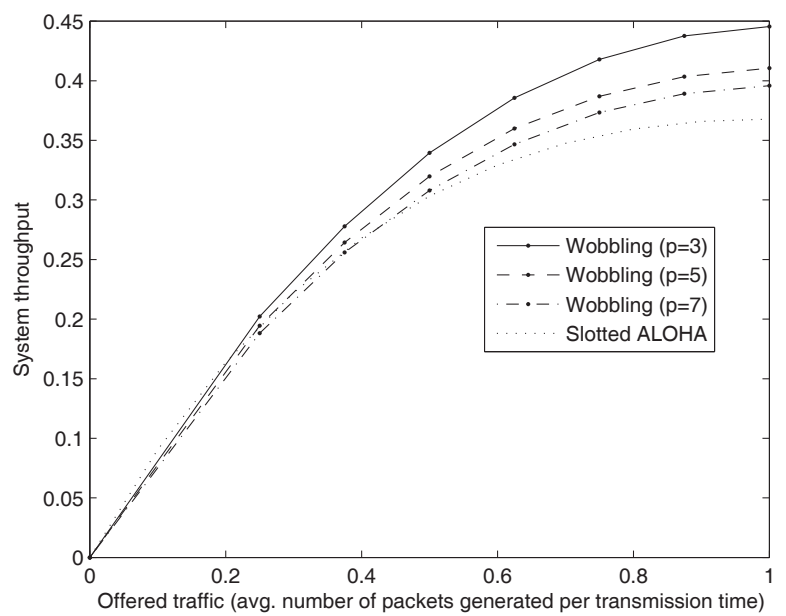

Figure 5: System throughput with respect to different offered channel traffic.

more energy-efficient. Besides, by the periodicity of wobbling sequence, there is a room to avoid some potential channel collisions. A simple predictive scheme is presented in the next section.

\section{Throughrut ENHANCEMENT}

One can find that a substantial amount of transmissions will be corrupted by packet collisions due to the nature of such an unsynchronized multiple access model. A simple listenand-avoid scheme is proposed in the following so as to enhance the effective transmission rates, particularly when the system throughput is a major concern. The idea can be integrated into the aforementioned protocol of wobbling sequence easily. From another point of view, the result also contributes to an improvement in the transmission power efficiency for energy-constraint sensor devices.

To avoid channel collisions with existing users, a new arrival is set to listen for a duration of the minimum common period before transmitting. After channel sensing, this user will exclude its predictively unnecessary transmission slots by comparing its protocol sequence with the existing transmission activities in order to avoid coming potential collisions. In other words, the new arrival (says, user $k$ ) will follow a modified wobbling sequence, $\widetilde{W^{(k)}}$, which is expressible as

$$
\widetilde{W}^{(k)}=W^{(k)}-W^{(k)} \bigcap\left(\bigcup_{j \neq k} W^{(j)}\right)
$$

where $W^{(j)}$ denotes the wobbling sequence employed by existing user $j$. This listen-and-avoid strategy can be manipulated individually in a distributed manner.

More importantly, such an avoidance could also help to eliminate its interference to existing active users based on the periodicity of wobbling sequences. It should be noted that the above strategy will be effective if users generally

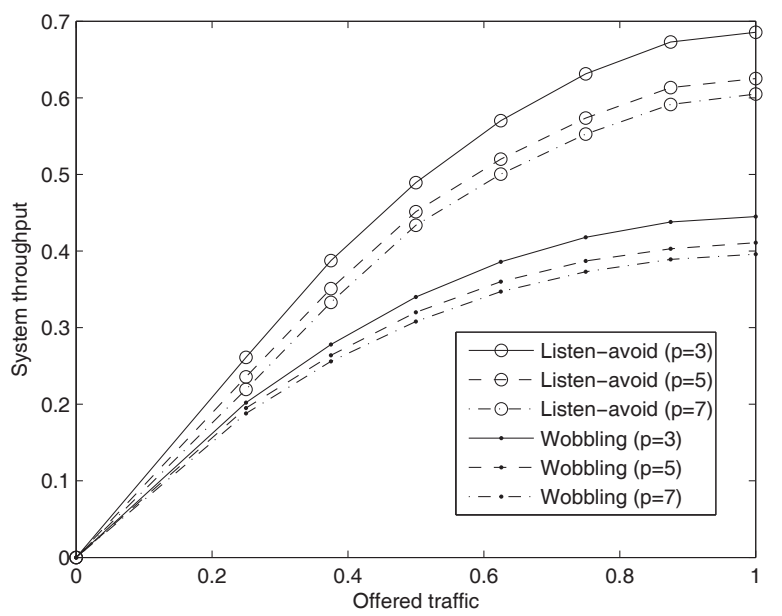

Figure 6: The system throughputs achieved with and without the collision avoidance mechanism are compared.

transmit in a relatively long time period as compared to their sequence period and the channel sensing duration. Otherwise, one should consider other techniques such as protocol sequence re-arrangement and precise contention resolution, if allowable. Besides, when a user finds its effective rate unsatisfied, it could be helpful to have an adaptive sequence re-scheduling for an improvement.

In simulation, we follow the same queuing model as that in Fig. 5, in which it is assumed that there are $p$ wobbling sequences available and each has a duty factor $1 / p$. However, the channel holding time of an active user here is assumed to have an exponential distribution with expected value of ten times of the sequence period $p^{4}$. We have investigated the system throughput in this model and compared the results achieved with and without the described collision avoidance mechanism respectively. As shown in Fig. 6, the system throughput enhancement is significant, especially in heavily loaded scenarios. The comparison indicates a substantial improvement in general due to the cooperative behavior among users. This consequently leads to an overall capacity gain. However, it should be noted that the effectiveness would generally depend on the system arrival and departure characteristics. It is worth of a further investigation.

\section{CONCLUSION}

In this paper, a simple and robust multiple access protocol which does not require complicated processing such as back-off algorithms or random number generation is introduced for wireless sensor and ad hoc networks. It offers an effective approach of distributed wireless access that can ensure the availability of a communication channel for any of the active users as long as the sum of duty factors does not exceed one. There cannot have enough collisions to completely block any particular user no matter how they are shifted with respect to one another. Simulation shows 
that both the individual and system throughputs have stable and satisfactory performance. Meanwhile, continuous channel listening during data reception can be avoided. In addition, a simple predictive collision avoidance scheme is proposed for the enhancement of system throughput. Note that it requires a channel listening in advance. The study here may also lead to other interesting schemes.

\section{APPENDIX}

Table 1: Multi-rate transmissions with sequences: two $1 / 3$, one $2 / 9$, two $1 / 27$. The requested rate $\operatorname{sum}=26 / 27$. The result is obtained from a number of $10^{5}$ runs.

\begin{tabular}{c|c|c|c}
\hline & Wobbling & Random & cf \\
\hline \hline mean sys. throughput & 0.437057 & 0.436812 & $26 / 27$ \\
\hline mean indiv. throughput & 0.087411 & 0.087362 & - \\
\hline max. indiv. throughput & 0.182899 & 0.172077 & $1 / 3$ \\
\hline min. indiv. throughput & 0.011584 & 0.007926 & $1 / 27$ \\
\hline
\end{tabular}

Table 2: Multi-rate transmissions with sequences: four $1 / 5$, one $4 / 25$, four $1 / 125$. The requested rate sum $=124 / 125$. The result is obtained from a number of $10^{5}$ runs.

\begin{tabular}{c|c|c|c}
\hline & Wobbling & Random & cf \\
\hline \hline mean sys. throughput & 0.429626 & 0.407403 & $124 / 125$ \\
\hline mean indiv. throughput & 0.047736 & 0.045267 & - \\
\hline max. indiv. throughput & 0.100687 & 0.084723 & $1 / 5$ \\
\hline min. indiv. throughput & 0.002486 & 0.002383 & $1 / 125$ \\
\hline
\end{tabular}

\section{REFERENCES}

[1] I. F. Akyildiz, W. Su, Y Sankarasubramaniam, and E. Cayirci, "A survey on sensor networks," IEEE Communications Magazine, vol. 40, no. 8, pp. 102-114, Aug. 2002.

[2] R. Jurdak, C. V. Lopes, and P. Baldi, "A survey, classification and comparative analysis of medium access control protocols for ad hoc networks," IEEE Commun. Surveys, vol. 6, no. 1, pp. 2-16, 2004.

[3] J. L. Massey and P. Mathys, "The collision channel without feedback," IEEE Trans. Information Theory, vol. 31, no. 2, pp. 192-204, 1985.

[4] J. Hui, "Multiple accessing for the collision channel without feedback," IEEE JSAC, vol. 2, no. 4, pp. 575-582, Jul. 1984.

[5] L. Gyorfi and I. Vajda, "Construction of protocol sequences for multiple-access collision channel without feedback," IEEE Trans. Information Theory, vol. 39, no. 5, pp. 1762-1765, 1992.

[6] Q. A. Nguyen, L. Gyorfi, and J. L. Massey, "Constructions of binary constant-weight cyclic codes and cyclically permutable codes," IEEE Trans. Inform. Theory, vol. 38, no. 3, pp. 940-949, 1992.

[7] G. Thomas, "Capacity of the wireless packet collision channel without feedback," IEEE Trans. Information Theory, May 2000.

[8] E. L. Titlebaum, "Time-frequency hop signals, part I: coding based upon the theory of linear congruences," IEEE Trans. Aerospace and Electronic Systems, vol. 17, no. 4, pp. 490-493, 1981.
[9] A. A. Shar and P. A. Davies, "Prime sequences: quasi-optimal sequences for OR channel code division multiplexing," IEE Electronics Letters, vol. 19, no. 21, pp. 888-890, 1983.

[10] P. R. Prucnal, M. A. Santoro, T. R. Fan, "Spread spectrum fiberoptic local area network using optical processing," IEEE J. Lightwave Tech., vol. 4, no. 5, pp. 547-554, 1986.

[11] W. C. Kwong, P. A. Perrier and P. R. Prucnal, "Performance comparison of asynchronous and synchronous code-division multiple-access techniques for fiber-optic local area networks," IEEE Trans. Communications, vol. 39, no. 11, pp. 1625-1634, 1991.

[12] C. S. Chen and W. S. Wong, "Bandwidth allocation for wireless multimedia systems with most regular sequences," IEEE Trans. Wireless Communications, vol. 4, no. 2, pp. 635-645, 2005.

[13] W. S. Wong, "New protocol sequences for random access channels without feedback," submitted for publication. 